\title{
Comparative Morphology of the Leaf Epidermis in Ligusticum (Apiaceae) from China
}

\author{
Jing Zhou ${ }^{*}$, Zhenwen Liu² \\ ${ }^{1}$ School of Pharmaceutical Sciences \& Yunnan Key Laboratory of Pharmacology for Nature Products, Kunming Medical \\ University, Kunming, China \\ ${ }^{2}$ Key Laboratory for Plant Diversity and Biogeography of East Asia, Kunming Institute of Botany, Chinese Academy of Sciences, \\ Kunming, China \\ Email: *zhoujing_apiaceae@163.com
}

How to cite this paper: Zhou, J. and Liu, Z.W. (2018) Comparative Morphology of the Leaf Epidermis in Ligusticum (Apiaceae) from China. American Journal of Plant Sciences, 9, 1105-1123.

https://doi.org/10.4236/ajps.2018.96084

Received: February 19, 2018

Accepted: May 14, 2018

Published: May 17, 2018

Copyright (C) 2018 by authors and Scientific Research Publishing Inc. This work is licensed under the Creative Commons Attribution International License (CC BY 4.0).

http://creativecommons.org/licenses/by/4.0/

\begin{abstract}
To investigate the taxonomic value of leaf epidermal characters in developing a classification for Ligusticum (Apiaceae), thirty-eight species (including a cultivar) of the genus were examined using both light and scanning electron microscopy. Our results show that the infrageneric classification of Ligusticum based on the characters of involucel bracteoles, mericarps, and pollen grains was not supported as few epidermal characters were relatively constant at section level. Evidence from leaf epidermis, similar to that from molecular and morphological data, supports the separation of Tilingia (i.e. L. ajanense and L. tachiroei) from Ligusticum. Ligusticopsis was found to be indistinguishable from Ligusticum in epidermal characters. Furthermore, results from epidermal analysis, together with that from cytological evidence, fruit morphology as well as molecular phylogenetics suggest that $L$. delavayi should be transferred out of Ligusticum.
\end{abstract}

\section{Keywords}

Leaf Epidermis, Ligusticum, Taxonomy, Umbelliferae (Apiaceae)

\section{Introduction}

The genus Ligusticum L., referred to the family Apiaceae, comprises 60 species widely distributed throughout Eurasia and North America. Of them, 40 species are known from China (of which 35 are being endemic), and most of these are distributed throughout the alpine belt of southwestern China (sub-alpine coniferous forests and forest edges, alpine scrub or meadows), with only a few species extending into northern China [1] [2]. Ligusticum exhibits a great diversity in 
the macro-morphological characters of leaf blades, bracteoles and mericarps, and is therefore one of the most difficult genera in the family Apiaceae for species delimitation, and the circumscription of Ligusticum has varied considerably [3] [4].

Drude [3] established three subgenera of Haloscia, Euligusticum and Pachypleurum in Ligusticum; Subsequently, Thellung [5], Schischkin [6] and Leute [7] added subgenus Mutellina, Pachypleuroides and Coristospermum successively. Even now, its relationships with putatively allied genera Cnidium Cuss., $\mathrm{Hy}_{\mathrm{y}}$ menidium DC., Pachypleurum Ledeb., Paraligusticum Tichom., Rupiphila Pimenov \& Lavrova, Selinum L., Tilingia Regel \& Tiling, and Ligusticopsis Leute are still not fully resolved [2]. The inclusion of Tilingia and Ligusticopsis within Ligusticum is particularly controversial [8] [9] [10]. Pu [1] arranged the species of Chinese Ligusticum into two sections: L. section Ligusticum L. and L. section Pinnatibracteola Pu based on the characters of involucel bracteoles, mericarps, the shape and aperture types of pollen grains. Doubt has already been cast on the rationality of this sectional system by our previous cytological study [11]. Many phylogenetic analyses on the Apiaceae family level have suggested that Ligusticum is a heterogeneous group as currently circumscribed [12]-[17], and that the Chinese Ligusticum species fall into three distant clades [18] [19]. Therefore, more morphological evidence is necessary before there can be further taxonomical revision of Ligusticum.

Leaf epidermal characteristics are of potential taxonomical importance [20] [21], of which features such as epidermal cells, types and arrangement of stomata, and surface ornamentation have been repeatedly proved well for taxonomy [22] [23] [24]. Sun et al. [25] have investigated the leaf epidermis morphology of Chinese species of Ligusticum, but this investigation was not comprehensive, with only 15 species examined. Thus, a more detailed observation of leaf epidermal morphology in the Chinese species of Ligusticum, through extensive sampling of taxa, is necessary. As part of a systemic study of this genus in China, we aim to investigate the epidermal features of 38 Chinese Ligusticum species, assess whether these epidermal features can provide information of taxonomic value and determine whether such information supports a sectional subdivision system in Chinese Ligusticum.

\section{Materials and Methods}

\section{Plant Materials}

For this study, thirty-six species of Ligusticum recognized in Flora of China (FOC) were investigated. Furthermore, two formally published species, L. moniliforme Peng \& Zhang and L. pseudodaucoides Peng \& Yin, which are not included by FOC were also considered to see if their epidermal characters are consistent with the genus (Table 1). Except fresh material collected from the field, other samples were taken from herbarium specimens in the Kunming Institute of Botany (KUN) and the Chengdu Institute of Biology (CDBI) (Table 1). All 
Table 1. Species, localities and vouchers for the leaf epidermal studies of Ligusticum (All specimens are deposited at KUN except for those labeled with CDBI).

\begin{tabular}{|c|c|c|}
\hline Taxon & Locality & Voucher \\
\hline \multicolumn{3}{|c|}{ Sect. Ligusticum } \\
\hline${ }^{\star}$ L. acuminatum Franch. & Maerkang, Sichuan & LZ1141 \\
\hline L. ajanense (Regel \& Tiling) Koso-Poljansky & Mt. Shirane, Japan & Miyoshi Fususe 45568 \\
\hline${ }^{\star}$ L. angelicifolium Franch. & Bomi, Xizang & $\mathrm{Z} 15120$ \\
\hline${ }^{\star}$ L. brachylobum Franch. & Lijiang, Yunnan & $\mathrm{Z} 1526$ \\
\hline L. delavayi Franch. & Heqing, Yunnan & LZ151006 \\
\hline L. discolor Ledeb. & Yili, Xinjiang & Zhangdy371 \\
\hline L. elatum (Edgew.) C. B. Clarke & Zuogong, Xizang & SunH-07ZX-1135 \\
\hline L. jeholense (Nakai \& Kitagawa) Nakai \& Kitagawa & Wanping, Hebei & YangCG 1691 \\
\hline L. kingdon-wardii $\mathrm{H}$. Wolff & Xiangcheng, Sichuan & Group of Qingzang 4022 (CDBI) \\
\hline L. litangense $\mathrm{Pu}$ & Litang, Sichuan & Z15157 \\
\hline L. littledalei Fedde ex H. Wolff & Chayu, Xizang & Z15109 \\
\hline L. nematophyllum (Pimenov \& Kljuykov) Pu \& Watson & Songpan, Sichuan & LZ1196 \\
\hline${ }^{\star}$ L. pteridophyllum Franch. & Xiangcheng, Sichuan & Z15177 \\
\hline L. reptans (Diels) H. Wolff & Luding, Sichuan & XuGH 26178 (CDBI) \\
\hline L. sinense Oliv. & Maerkang, Sichuan & LZ1169 \\
\hline L. sinense cv. Chuanxiong Qiu et al. & Guangnan, Yunnan & LZ20160607 \\
\hline L. tachiroei (Franch. \& Savat.) Hiroe \& Constance & Mt. Baihua, Beijing & L04 \\
\hline${ }^{\star}$ L. tenuisectum $\mathrm{H}$. de Boissieu & Mt. Jinfo, Chongqing & ZW20090917 \\
\hline L. tenuissimum $\mathrm{H}$. de Boissieu & Neiqiu, Hebei & LiuXY 747 \\
\hline L. thomsonii C. B. Clarke & Yushu, Qinghai & Liujq-QLS-TXM-089 \\
\hline L. yanyuanense $\mathrm{Pu}$ & Yanyuan, Sichuan & Group of Qingzang 12179 \\
\hline \multicolumn{3}{|c|}{ Sect. Pinnatibracteola } \\
\hline${ }^{\star}$ L. capillaceum $\mathrm{H}$. Wolff & Xiangcheng, Sichuan & Z15182 \\
\hline${ }^{\star}$ L. daucoides (Franch.) Franch. & Shangrila,Yunnan & Z15194 \\
\hline${ }^{\star} L$. franchetii $\mathrm{H}$. de Boissieu & Muli, Sichuan & CaoYL \& YaoJ 284 (CDBI) \\
\hline${ }^{\star}$ L. hispidum (Franch.) H. Wolff & Lijiang, Yunnan & $\mathrm{Z} 1508$ \\
\hline L. involucratum Franch. & Lijiang, Yunnan & Z1509 \\
\hline${ }^{\star}$ L. likiangense $(\mathrm{H}$. Wolff) $\mathrm{Pu} \&$ Watson & Heqing, Yunnan & LZ151005 \\
\hline${ }^{\star}$ L. multivittatum Franch. & Xiangcheng, Sichuan & $\mathrm{Z} 15172$ \\
\hline${ }^{\star}$ L. oliverianum $(\mathrm{H}$. de Boissieu) Shan & Kangding, Sichuan & LZ1107 \\
\hline${ }^{\star}$ L. rechingerianum (Leute) Shan $\& \mathrm{Pu}$ & Deqin, Yunnan & ZhouZK-07ZX-0424 \\
\hline${ }^{\star}$ L. scapiforme $\mathrm{H}$. Wolff & Muli, Sichuan & LZ1036 \\
\hline L. sikiangense Hiroe & Lugu Lake, Yunnan & LZ1022 \\
\hline \multicolumn{3}{|c|}{ Species not included in Pu's division } \\
\hline L. mairei Hiroe & Qiaojia, Yunnan & HP5121 \\
\hline L. moniliforme Peng \& Zhang & Gannan, Gansu & LiuJQ-GN-2011-269 \\
\hline L. mucronatum (Schrenk) Leute & Zhaosu, Xinjiang & Shengtian 957 \\
\hline L. nullivittatum $(\mathrm{Fu}) \mathrm{Pu} \&$ Watson & Zhouzhi, Shanxi & ZhangXM 135 \\
\hline L. pseudodaucoides Peng \&Yin & Jingdong, Yunnan & HP2579 \\
\hline L. weberbauerianum Fedde ex H. Wolff & Yajiang, Sichuan & FeiY \& LiLQ 2969 \\
\hline
\end{tabular}


materials were identified by the first author using published keys. Mature leaves were investigated under light microscopy (LM) and scanning electron microscopy (SEM). To check the constancy of epidermal structure, at least five slides were made from different parts of a single leaf for each species. Materials for LM study were boiled in distilled water for 20 minutes before being macerated in $75 \% \mathrm{NaClO}$. Pieces of leaf epidermis were stained with Safranin solution (1\%), and then dehydrated in an ethanol series (from 75\% to 100\%) before being mounted in Canada balsam. Materials for SEM were directly attached to stubs without any treatment. After gold sputtering, the specimens were examined and photographed under a Hitachi S-4800. Stomatal terminology was based on the classification proposed by Baranova [21] [26] and Wilkinson [27].

\section{Results}

Leaf epidermal features under LM and SEM listed in Table $2 \&$ Table 3, corresponding to Figures 1-6. Other figures are provided as Supplementary material (Figures S1-S3).

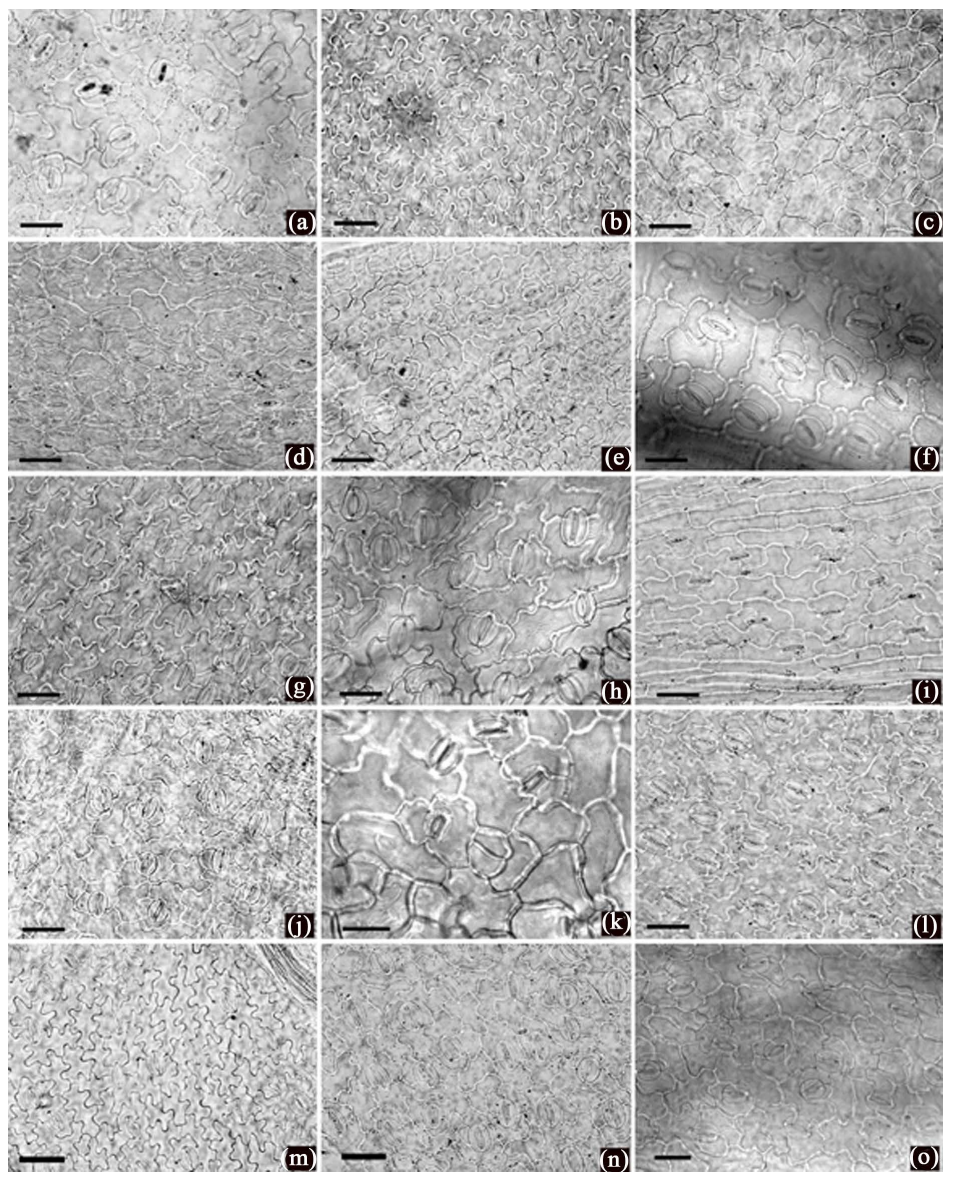

Figure 1. Characteristics of epidermal cells from Ligusticum species (light microscopy), all abaxial. (a) L. daucoides; (b) L. pteridophyllum; (c) L. litangense; (d) L. tachiroei; (e) L. hispidum; (f) L. nematophyllum; (g) L. sinense; (h) L. involucratum; (i) L. delavayi; (j) L. oliverianum; (k) L. likiangense; (l) L. littledalei; (m) L. acuminatum; (n) L. angelicifolium; (o) L. scapiforme. Scale bar $50 \mu \mathrm{m}$. 
Table 2. Leaf epidermal characters of Ligusticum under LM (surface view).

\begin{tabular}{|c|c|c|c|c|c|c|c|c|}
\hline \multirow[b]{2}{*}{ Taxon } & \multicolumn{2}{|c|}{ Adaxial Epidermis } & \multicolumn{5}{|c|}{ Abaxial Epidermis } & \multirow[b]{2}{*}{ Figure } \\
\hline & $\begin{array}{l}\text { Stomatal } \\
\text { apparatus }\end{array}$ & $\begin{array}{l}\text { Pattern of } \\
\text { anticlinal } \\
\text { walls }\end{array}$ & $\begin{array}{c}\text { Shape of } \\
\text { ordinary cells }\end{array}$ & $\begin{array}{l}\text { Pattern of } \\
\text { anticlinal } \\
\text { walls }\end{array}$ & Stomatotype & $\begin{array}{l}\text { Stomata } \\
\text { index }(\%)\end{array}$ & $\begin{array}{c}\text { Size of } \\
\text { stomata }(\mu \mathrm{m})^{2}\end{array}$ & \\
\hline \multicolumn{9}{|l|}{ Sect. Ligusticum } \\
\hline L. acuminatum & A & Sinuous & Irr & Sinuous & Ano & 18.6 & $13.37 \times 7.47$ & $1(\mathrm{~m}), \mathrm{S} 1(\mathrm{~m})$ \\
\hline L. ajanense & A & Str-Arc & Irr & Repand & Ani & 25.8 & $24.18 \times 12.39$ & $2(\mathrm{o}), \mathrm{S} 2(\mathrm{o})$ \\
\hline L. angelicifolium & A & Repand & Irr & Repand & Ani & 24.4 & $22.96 \times 11.69$ & $1(\mathrm{n}), \mathrm{S} 1(\mathrm{n})$ \\
\hline L. brachylobum & $\mathrm{P}$ & Repand & Irr & Repand & Ani & 28.6 & $21.49 \times 10.95$ & $2(\mathrm{f}), \mathrm{S} 2(\mathrm{f})$ \\
\hline L. delavayi & $\mathrm{P}$ & Repand & Irr + rectangular & Repand & Ani & 18.0 & $15.37 \times 9.26$ & 1(i), S1(i) \\
\hline L. discolor & $\mathrm{P}$ & Sinuous & Irr & Sinuous & Ano & 23.1 & $21.19 \times 10.00$ & 2(1), S2(l) \\
\hline L. elatum & $\mathrm{P}$ & Str-Arc & Pol & Str-Arc & Ani & 17.9 & $16.13 \times 10.67$ & $3(b), S 3(b)$ \\
\hline L. jeholense & A & Sinuous & Irr & Sinuous & Ano & 20.6 & $17.29 \times 9.65$ & $2(\mathrm{~m}), \mathrm{S} 2(\mathrm{~m})$ \\
\hline L. kingdon-wardii & A & Str-Arc & Irr & Sinuous & Ano & 18.2 & $25.09 \times 14.15$ & $3(d), S 3(d)$ \\
\hline L. litangense & $\mathrm{P}$ & Str-Arc & Pol & Str-Arc & Ani & 23.6 & $19.85 \times 10.15$ & $1(\mathrm{c}), \mathrm{S} 1(\mathrm{c})$ \\
\hline L. littledalei & A & Repand & Irr & Sinuous & Ano & 25.0 & $16.82 \times 10.71$ & $1(1), S 1(1)$ \\
\hline L. nematophyllum & A & Repand & Irr & Repand & Ani & 21.1 & $24.00 \times 12.91$ & $1(\mathrm{f}), \mathrm{S} 1(\mathrm{f})$ \\
\hline L. pteridophyllum & A & Sinuous & Irr & Sinuous & Ano & 23.5 & $18.46 \times 12.69$ & 1(b), S1(b) \\
\hline L. reptans & $\mathrm{A}$ & Str-Arc & Pol & Str-Arc & Ani & 30.1 & $18.90 \times 9.59$ & $3(\mathrm{c}), \mathrm{S} 3(\mathrm{c})$ \\
\hline L. sinense & A & Sinuous & Irr & Sinuous & Ano & 28.4 & $15.24 \times 5.95$ & $1(\mathrm{~g}), \mathrm{S} 1(\mathrm{~g})$ \\
\hline L. sinense cv. Chuanxiong & A & Repand & Irr & Sinuous & Ano & 19.3 & $23.35 \times 14.65$ & $3(\mathrm{~h}), \mathrm{S} 3(\mathrm{~h})$ \\
\hline L. tachiroei & A & Str-Arc & Irr & Repand & Ani & 15.4 & $15.92 \times 7.50$ & $1(\mathrm{~d}), \mathrm{S} 1(\mathrm{~d})$ \\
\hline L. tenuisectum & A & Repand & Irr & Sinuous & Ani & 18.9 & $29.09 \times 9.48$ & 2(e), S2(e) \\
\hline L. tenuissimum & A & Str-Arc & Pol & Str-Arc & Ani & 15.9 & $18.82 \times 8.97$ & $2(\mathrm{~g}), \mathrm{S} 2(\mathrm{~g})$ \\
\hline L. thomsonii & $\mathrm{P}$ & Str-Arc & Irr & Repand & Ani & 19.8 & $17.05 \times 10.21$ & $2(\mathrm{j}), \mathrm{S} 2(\mathrm{j})$ \\
\hline L. yanyuanense & $\mathrm{P}$ & Str-Arc & Pol & Str-Arc & Ani & 23.4 & $22.46 \times 13.28$ & 2(h), S2(h) \\
\hline \multicolumn{9}{|l|}{ Sect. Pinnatibracteola } \\
\hline L. capillaceum & $\mathrm{P}$ & Repand & Irr & Repand & Ani & 20.6 & $14.25 \times 10.38$ & $2(c), S 2(c)$ \\
\hline L. daucoides & $\mathrm{P}$ & Repand & Irr & Repand & Ani & 17.6 & $17.41 \times 11.53$ & 1(a), S1(a) \\
\hline L. franchetii & A & Repand & Irr & Sinuous & Ano & 25.2 & $15.51 \times 9.31$ & $3(\mathrm{e}), \mathrm{S} 3(\mathrm{e})$ \\
\hline L. hispidum & $\mathrm{P}$ & Repand & Irr & Repand & Ani & 11.0 & $13.81 \times 8.21$ & $1(\mathrm{e}), \mathrm{S} 1(\mathrm{e})$ \\
\hline L. involucratum & $\mathrm{P}$ & Str-Arc & Irr & Repand & Ani & 17.7 & $20.76 \times 13.03$ & 1(h), S1(h) \\
\hline L. likiangense & $\mathrm{P}$ & Str-Arc & Irr & Repand & Ani & 19.0 & $21.22 \times 12.44$ & $1(\mathrm{k}), \mathrm{S} 1(\mathrm{k})$ \\
\hline L. multivittatum & $\mathrm{P}$ & Repand & Irr & Sinuous & Ano & 17.0 & $13.25 \times 9.67$ & $2(\mathrm{~d}), \mathrm{S} 2(\mathrm{~d})$ \\
\hline L. oliverianum & $\mathrm{P}$ & Sinuous & Irr & Sinuous & Ani & 17.3 & $15.90 \times 10.86$ & $1(\mathrm{j}), \mathrm{S} 1(\mathrm{j})$ \\
\hline L. rechingerianum & $\mathrm{P}$ & Str-Arc & Pol & Str-Arc & Ani & 12.8 & $13.91 \times 10.14$ & $3(\mathrm{~g}), \mathrm{S} 3(\mathrm{~g})$ \\
\hline L. scapiforme & $\mathrm{P}$ & Repand & Irr & Repand & Ani & 24.4 & $14.59 \times 9.42$ & 1(o), S1(o) \\
\hline L. sikiangense & $\mathrm{P}$ & Repand & Irr & Repand & Ani & 18.5 & $19.74 \times 10.81$ & $2(\mathrm{a}), \mathrm{S} 2(\mathrm{a})$ \\
\hline \multicolumn{9}{|l|}{$\begin{array}{c}\text { Species not } \\
\text { included in Pu's division }\end{array}$} \\
\hline L. mairei & $\mathrm{P}$ & Str-Arc & Pol & Str-Arc & Ani & 19.4 & $25.17 \times 15.67$ & 2(b), S2(b) \\
\hline L. moniliforme & A & Sinuous & Irr & Sinuous & Ano & 28.3 & $41.22 \times 20.49$ & $3(\mathrm{f}), \mathrm{S} 3(\mathrm{f})$ \\
\hline L. mucronatum & $\mathrm{P}$ & Str-Arc & Irr & Repand & Ani & 14.9 & $18.82 \times 13.16$ & 2(i), S2(i) \\
\hline L. nullivittatum & A & Sinuous & Irr & Sinuous & Ano & 27.4 & $19.75 \times 9.88$ & $2(\mathrm{k}), \mathrm{S} 2(\mathrm{k})$ \\
\hline L. pseudodaucoides & A & Str-Arc & Pol & Str-Arc & Ani & 25.6 & $21.32 \times 13.09$ & $3(\mathrm{a}), \mathrm{S} 3(\mathrm{a})$ \\
\hline L. weberbauerianum & A & Str-Arc & Pol & Str-Arc & Ani & 11.7 & $18.89 \times 12.36$ & $2(\mathrm{n}), \mathrm{S} 2(\mathrm{n})$ \\
\hline
\end{tabular}

A: Absent; P: Present; Pol: Polygonal; Irr: Irregular; Str-Arc: Straight to arched; Ani: Anisocytic; Ano: Anomocytic. 
Table 3. Leaf epidermal characters of Ligusticum under SEM (surface view).

\begin{tabular}{|c|c|c|c|c|c|}
\hline \multirow[b]{2}{*}{ Taxon } & \multicolumn{4}{|c|}{ Abaxial epidermis } & \multirow[b]{2}{*}{ Figure } \\
\hline & $\begin{array}{l}\text { Outline of the pair } \\
\text { of guard cells }\end{array}$ & $\begin{array}{l}\text { Inner margin of } \\
\text { outer stomatal rim }\end{array}$ & $\begin{array}{l}\text { Cuticular membrane } \\
\text { ornamentation }\end{array}$ & $\begin{array}{c}\text { Wax } \\
\text { ornamentation }\end{array}$ & \\
\hline \multicolumn{6}{|l|}{ Sect. Ligusticum } \\
\hline L. acuminatum & $\mathrm{NE}$ & Smooth & Striate & Invisible & $4(\mathrm{~m})$ \\
\hline L. ajanense & Ell & Smooth & Wrinkled \& Striate & Scales & $5(0)$ \\
\hline L. angelicifolium & Ell & Smooth & Striate & Invisible & $4(\mathrm{n})$ \\
\hline L. brachylobum & $\mathrm{NE}$ & Smooth & Striate & Invisible & $5(\mathrm{f})$ \\
\hline L. delavayi & Ell & Smooth & Smooth & Invisible & $4(\mathrm{i})$ \\
\hline L. discolor & Ell & Erose & Striate & Flackes & $5(1)$ \\
\hline L. elatum & Ell & Smooth & Striate & Invisible & $6(\mathrm{~b})$ \\
\hline L. jeholense & Ell & Smooth & Sparse radiating Striate & Invisible & $5(\mathrm{~m})$ \\
\hline L. kingdon-wardii & $\mathrm{NE}$ & Smooth & Striate & Invisible & $6(\mathrm{~d})$ \\
\hline L. litangense & Ell & Smooth & Striate & Invisible & $4(\mathrm{c})$ \\
\hline L. littledalei & Ell & Smooth & Striate & Invisible & $4(1)$ \\
\hline L. nematophyllum & Ell & Smooth & Striate & Invisible & $4(\mathrm{f})$ \\
\hline L. pteridophyllum & WE & Nearly Smooth & Striate & Invisible & $4(\mathrm{~b})$ \\
\hline L. reptans & $\mathrm{NE}$ & Smooth & Striate & Invisible & $6(c)$ \\
\hline L. sinense & $\mathrm{NE}$ & Erose & Striate & Invisible & $4(\mathrm{~g})$ \\
\hline L. sinense cv. Chuanxiong & $\mathrm{NE}$ & Smooth & Sparse radiating Striate & Invisible & $6(\mathrm{~h})$ \\
\hline L. tachiroei & $\mathrm{NE}$ & Erose & Striate & Invisible & $4(\mathrm{~d})$ \\
\hline L. tenuisectum & Ell & Smooth & Striate & Invisible & $5(\mathrm{e})$ \\
\hline L. tenuissimum & $\mathrm{NE}$ & Erose & Striate & Invisible & $5(\mathrm{~g})$ \\
\hline L. thomsonii & Ell & Erose & Striate & Invisible & $5(\mathrm{j})$ \\
\hline L. yanyuanense & Ell & Erose & Sparse radiating Striate & Parcels & $5(\mathrm{~h})$ \\
\hline \multicolumn{6}{|l|}{ Sect. Pinnatibracteola } \\
\hline L. capillaceum & Ell & Smooth & Striate & Invisible & $5(\mathrm{c})$ \\
\hline L. daucoides & Ell & Erose & Wrinkled & Scales & $4(\mathrm{a})$ \\
\hline L. franchetii & $\mathrm{NE}$ & Erose & Striate & Invisible & $6(\mathrm{e})$ \\
\hline L. hispidum & Ell & Smooth & Wrinkled & Flacks & $4(\mathrm{e})$ \\
\hline L. involucratum & Ell & Smooth & Radiating Striate & Invisible & $4(\mathrm{~h})$ \\
\hline L. likiangense & Ell & Smooth & Sparse radiating Striate & Invisible & $4(\mathrm{k})$ \\
\hline L. multivittatum & Ell & Smooth & Striate & Invisible & $5(\mathrm{~d})$ \\
\hline L. oliverianum & Ell & Erose & Striate & Invisible & $4(\mathrm{j})$ \\
\hline L. rechingerianum & Rou & Erose & Smooth & Scales & $6(\mathrm{~g})$ \\
\hline L. scapiforme & Ell & Erose & Wrinked \& Striate & Invisible & $4(\mathrm{o})$ \\
\hline L. sikiangense & Ell & Smooth & Striate & Invisible & $5(\mathrm{a})$ \\
\hline \multicolumn{6}{|c|}{ Species not included in Pu's division } \\
\hline L. mairei & Ell & Smooth & Striate & Invisible & $5(\mathrm{~b})$ \\
\hline L. moniliforme & Ell & Smooth & Striate & Invisible & $6(f)$ \\
\hline L. mucronatum & Ell & Erose & Striate & Invisible & $5(\mathrm{i})$ \\
\hline L. nullivittatum & Ell & Smooth & Concentric Striate & Invisible & $5(\mathrm{k})$ \\
\hline L. pseudodaucoides & Ell & Smooth & Wrinkled \& Striate & Invisible & $6(a)$ \\
\hline L. weberbauerianum & Ell & Smooth & Concentric Striate & Invisible & $5(\mathrm{n})$ \\
\hline
\end{tabular}

Ell: Elliptical; NE: Narrowly Elliptical; WE: Widely Elliptical; Rou: Roundish. 


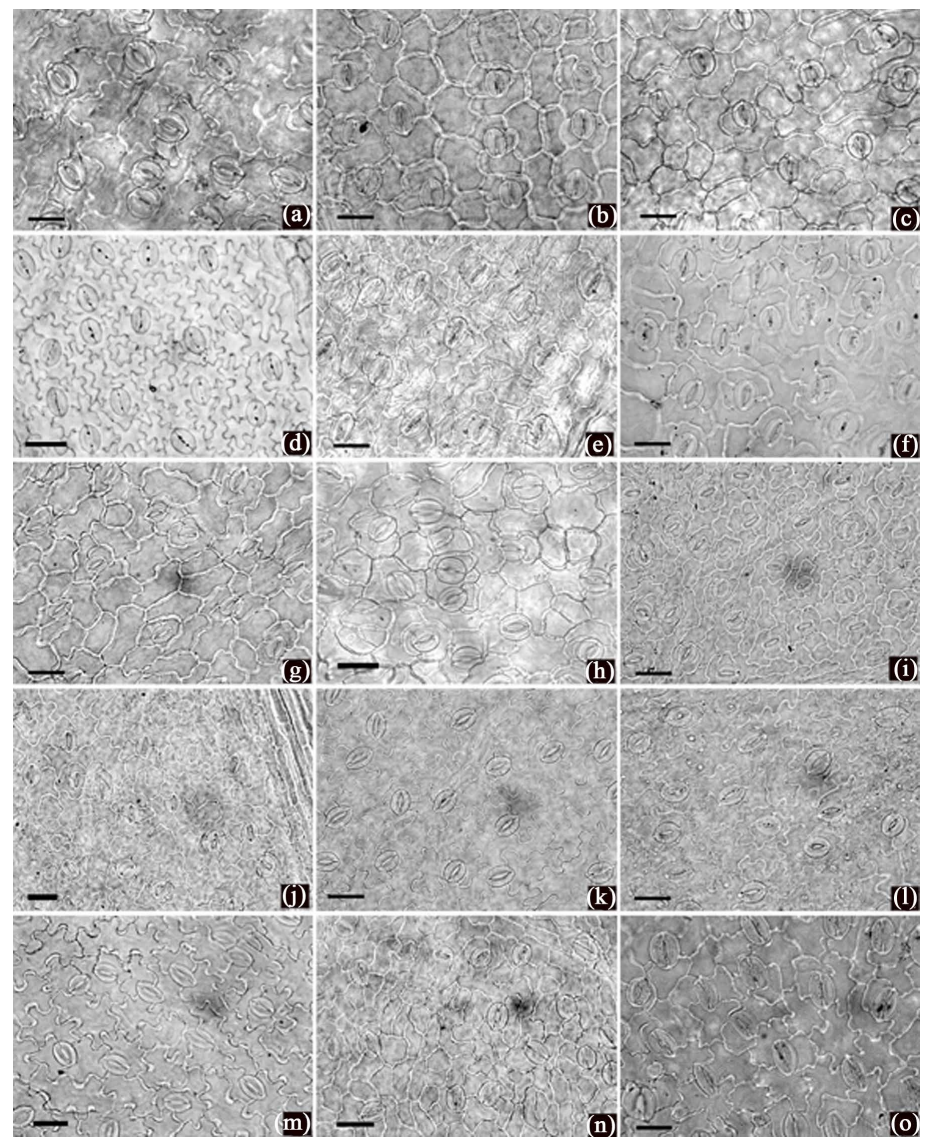

Figure 2. Characteristics of epidermal cells from Ligusticum species (light microscopy), all abaxial. (a) L. sikiangense; (b) L. maire; (c) L. capillaceum; (d) L. multivittatum; (e) L. tenuisectum; (f) L. brachylobum; (g) L. tenuissimum; (h) L. yanyuanense; (i) L. mucronatum; (j) L. thomsonii; (k) L. nullivittatum; (l) L. discolor, (m) L. jeholense; (n) L. weberbauerianum; (o) L. ajanense. Scale bar $50 \mu \mathrm{m}$.

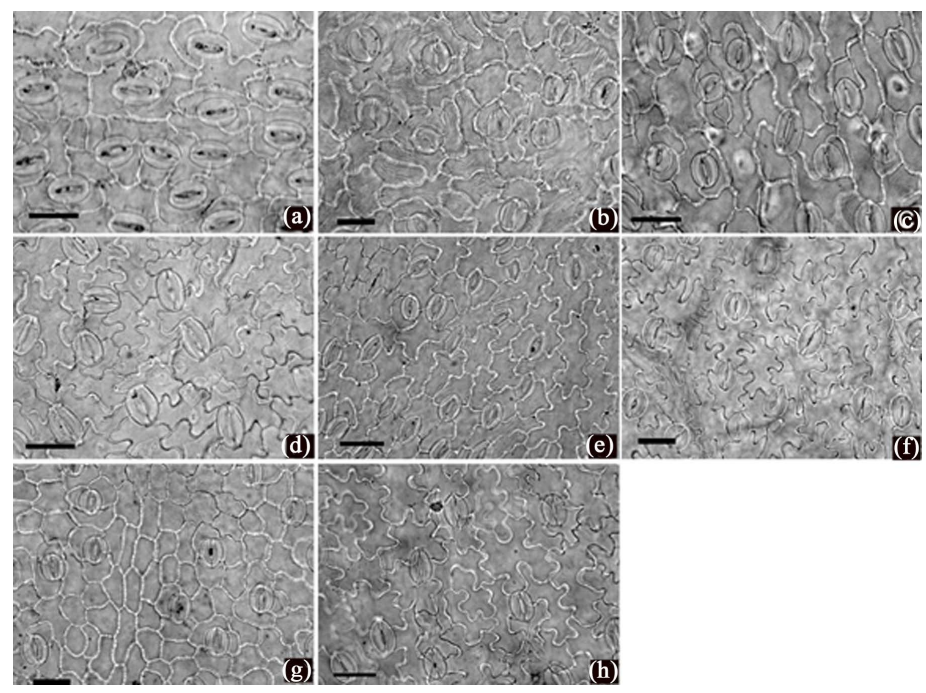

Figure 3. Characteristics of epidermal cells from Ligusticum species (light microscopy), all abaxial. (a) L. pseudodaucoides; (b) L. elatum; (c) L. reptans; (d) L. kingdon-wardii; (e) L. franchetii; (f) L. moniliforme; (g) L. rechingerianum; (h) L. sinense cv. Chuanxiong. Scale bar $50 \mu \mathrm{m}$. 

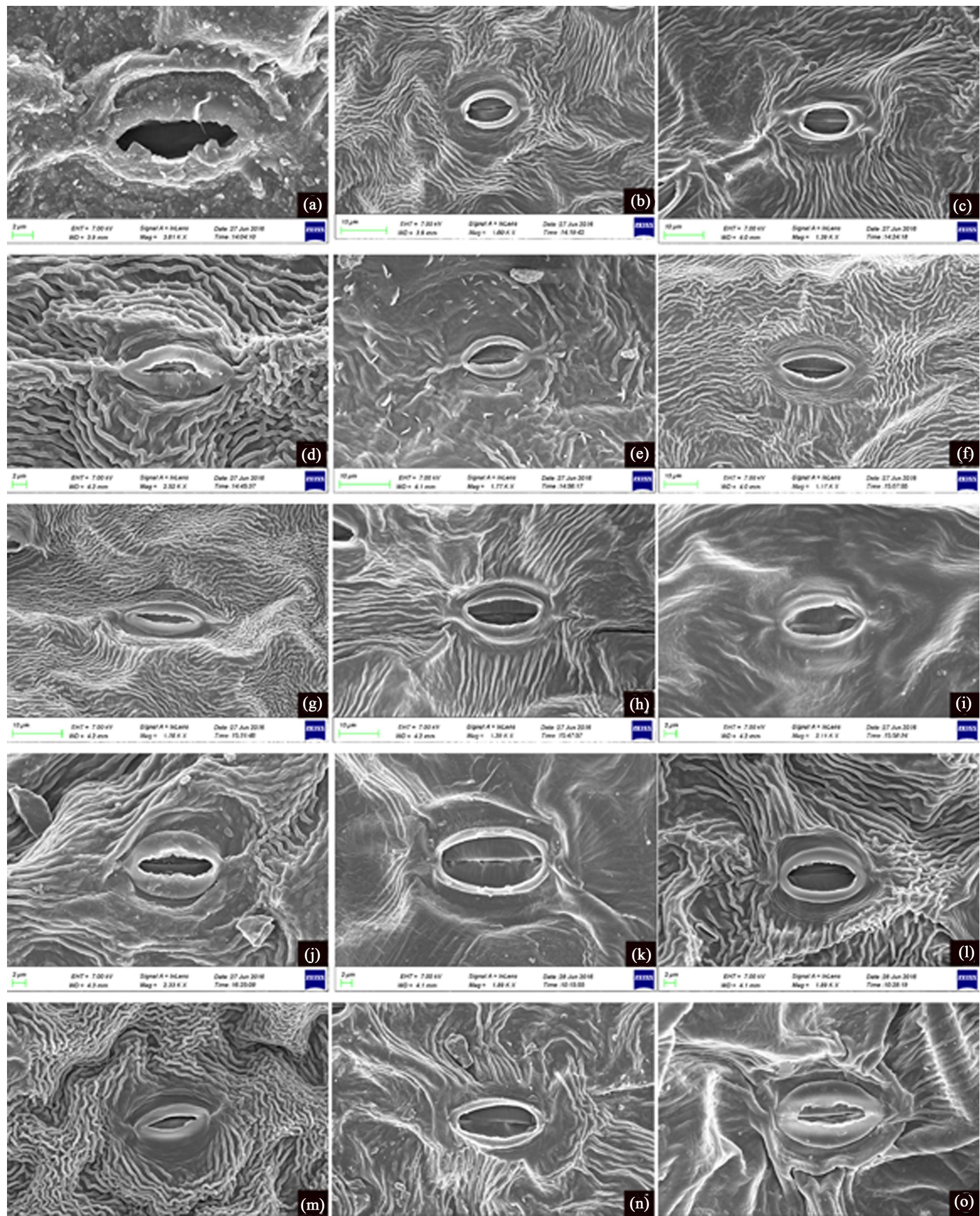

Figure 4. Characteristics of epidermal cells from Ligusticum species (scanning electron microscopy), all abaxial. (a) L. daucoides, (b) L. pteridophyllum; (c) L. litangense; (d) L. tachiroei; (e) L. hispidum; (f) L. nematophyllum; (g) L. sinense; (h) L. involucratum; (i) L. delavayi; (j) L. oliverianum; (k) L. likiangense; (l) L. littledalei; (m) L. acuminatum; (n) L. angelicifolium; (o) L. scapiforme. Scale bar $20 \mu \mathrm{m}$. 

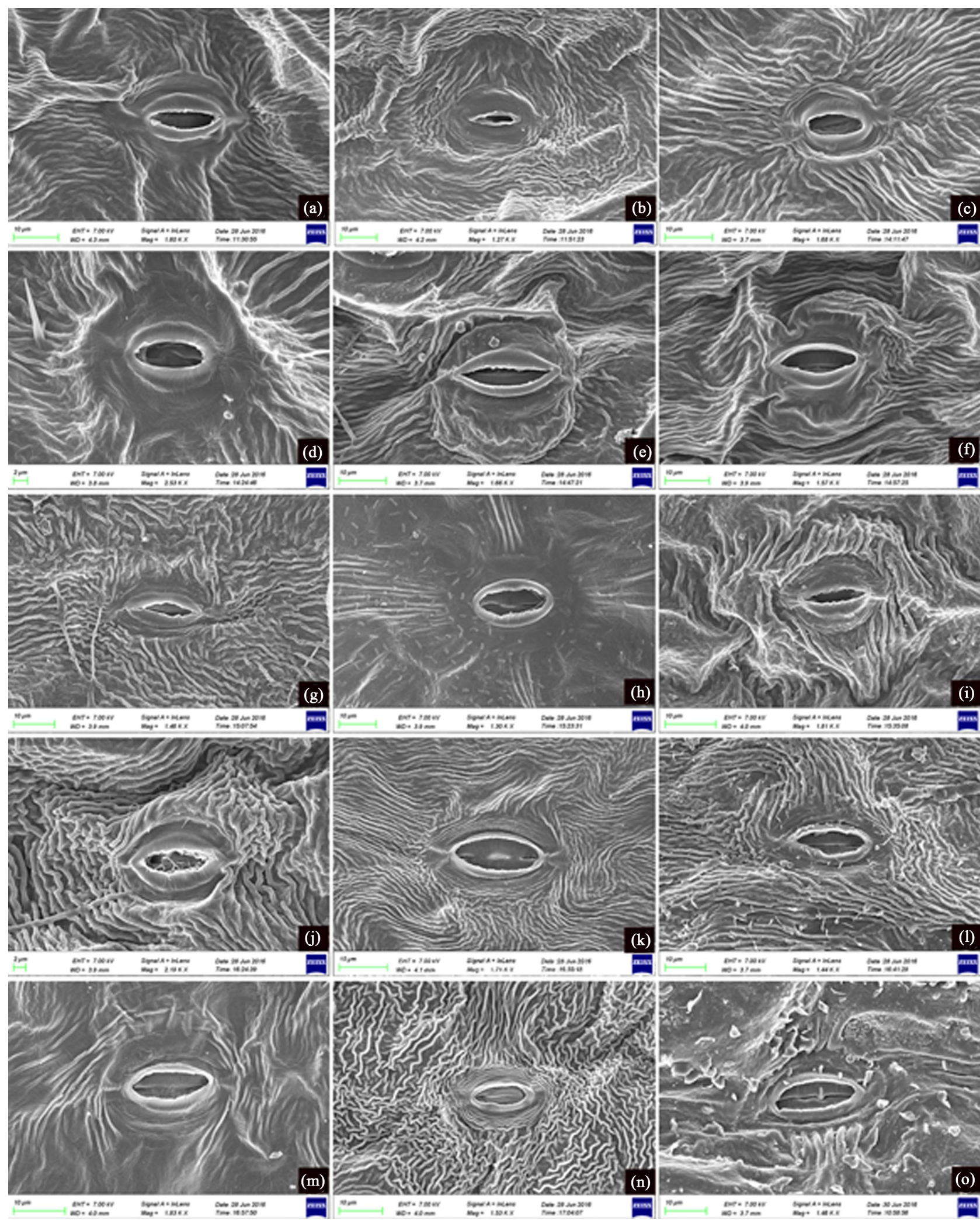

Figure 5. Characteristics of epidermal cells from Ligusticum species (scanning electron microscopy), all abaxial. (a) L. sikiangense; (b) L. mairei; (c) L. capillaceum; (d) L. multivittatum; (e) L. tenuisectum; (f) L. brachylobum; (g) L. tenuissimum; (h) L. yanyuanense; (i) L. mucronatum; (j) L. thomsonii; (k) L. nullivittatum; (l) L. discolor, (m) L. jeholense; (n) L. weberbauerianum; (o) L. ajanense. Scale bar $20 \mu \mathrm{m}$. 

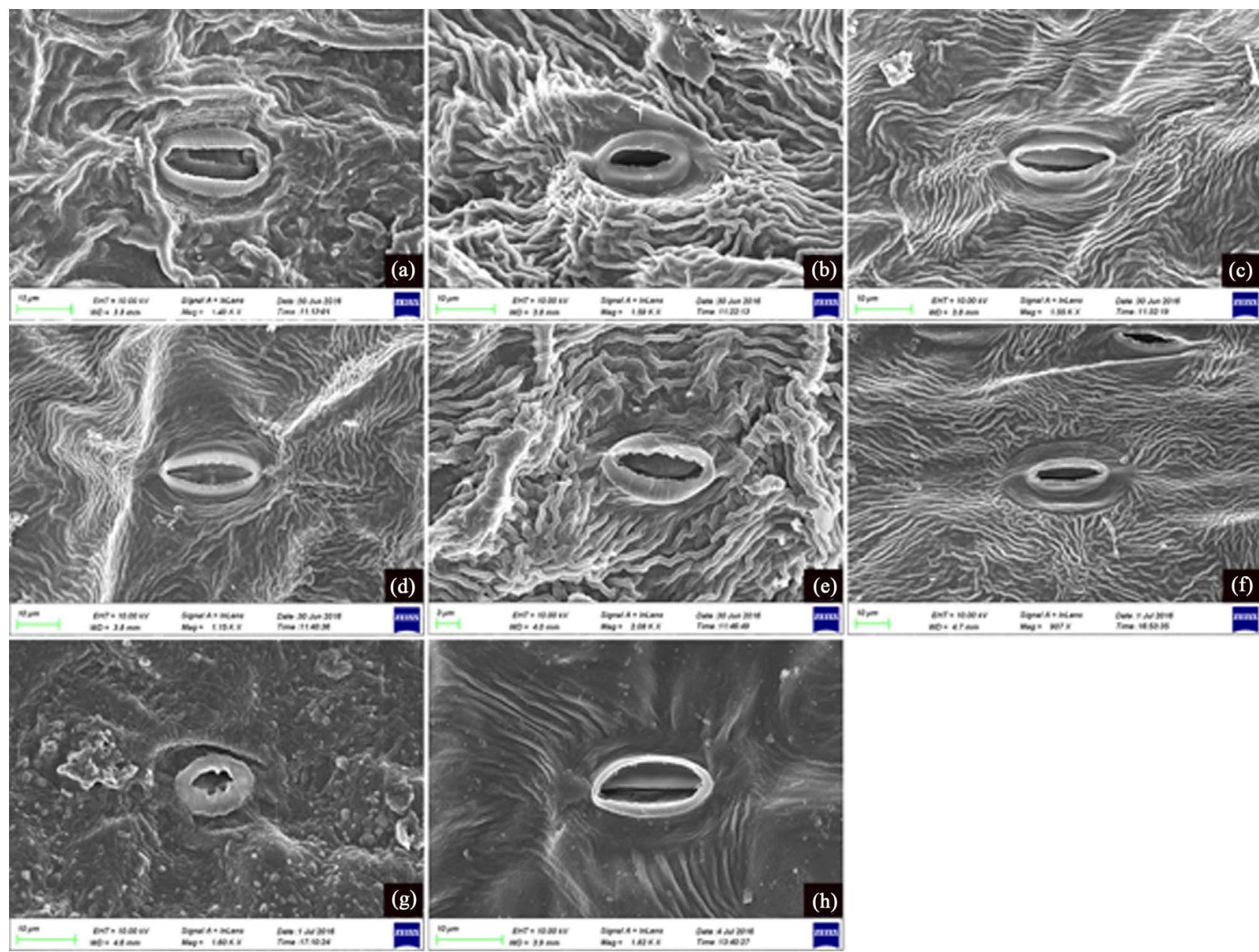

Figure 6. Characteristics of epidermal cells from Ligusticum species (scanning electron microscopy), all abaxial. (a) L. pseudodaucoides; (b) L. elatum; (c) L. reptans; (d) L. kingdon-wardii; (e) L. franchetii; (f) L. moniliforme; (g) L. rechingerianum; (h) L. sinense cv. Chuanxiong. Scale bar $20 \mu \mathrm{m}$.

\subsection{Characteristics of the Leaf Epidermis under LM}

The form of epidermal cells in Ligusticum as seen under LM was usually irregular or polygonal (rarely rectangular in L. delavayi, Figure 1(i) and Figure S1(i)), with anticlinal walls straight to arched, repand or sinuous (Table 2). The pattern of anticlinal cells may vary between different species and also between the adaxial and abaxial epidermis of a given species. In about $2 / 3$ of the species studied, the anticlinal cells of both sides were consistent with straight to arched walls or repand and sinuous walls, while in the other 12 species, the anticlinal walls varied between the adaxial and abaxial surfaces (Table 2). Stomata were present on both surfaces in 19 of the observed species (Table 2). The patters of the stomata were anisocytic or anomocytic, inconsistent with Sun et al. [25] in which four types of stomata were found. The stomatal index ranged from $11.0 \%$ in L. hispidum (Figure 1(e) and Figure S1(e)), up to 30.1\% in L. reptans (Figure 3(c) and Figure S3(c)). Particularly, stomatas observed were usually closed, indicating that Ligusticum is relatively suitable to arid habitats. 


\subsection{Characteristics of the Leaf Epidermis under SEM}

The outlines of the pair of guard cells were elliptical, narrowly elliptical, and roundish. The inner margins of the outer stomatal rim were observed to be of two types of smooth or nearly smooth, and erose (Table 3). The cuticular membrane ornamentations were striate, wrinkled and smooth. The striate type was found in most species investigated, with some variations discovered in a few taxa: a sparse radiating striate cuticular membrane ocurred in L. jeholense (Figure $5(\mathrm{~m})$ ), L. sinense cv. Chuanxiong (Figure 6(h)), L. yanyuanense (Figure 5(h)) and L. likiangense (Figure 4(k)); the membrane of L. involucratum (Figure $4(\mathrm{~h})$ ) was of a radiating striate type; and a concentric striate membrane was found in both $L$. weberbauerianum (Figure 5(n)) and L. nullivittatum (Figure 5(k)). A wrinkled type was present in both L. daucoides (Figure 4(a)) and L. hispidum (Figure 4(e)). L. delavayi (Figure 4(i)) and L. rechingerianum (Figure 6(g)) had smooth ornamentations. The cuticular membranes of the remaining species were of a mixture of two types. Both wrinkled and striate membranes were present in L. ajanense (Figure 5(o)), L. scapiforme (Figure 4(o)) and L. pseudodaucoides (Figure 6(a)).

Wax ornamentation was absent in 32 species, while the remaining six had wax scales or other ornamentations. Scales occurred in three species: Ligusticum ajanense (Figure 5(0)), L. daucoides (Figure 4(a)) and L. rechingerianum (Figure 6(g)). L. discolor (Figure 5(1)) and L. hispidum (Figure 4(e)) had flacks, while parcels appeared in $L$. yanyuanense (Figure 5(h)).

\section{Discussion}

It was observed that most species has its own unique combination of leaf epidermal features, making the characters taxonomically useful. In our analysis, epidermal characters with potential systematic values are compared with previous phylogenetic results, and morphological traits such as fruit are also considered. Pu [1] (1991) arranged the species of Chinese Ligusticum into two sections: section Ligusticum with bracteoles linear or lanceolate, entire, and section Pinnatibracteola with bracteoles 1 - 3-pinnatisect or 2 - 3-lobed at the apex. In our study, 21 species of sect. Ligusticum and 11 species of sect. Pinnatibracteola were included. As indicated in Table 2 \& Table 3, no differences were detected in epidermal characters under LM and SEM between these two sections, both sections having irregular and polygonal epidermal cells, straight to arched, sinuous and repand anticlinal walls, anisocytic and anomocytic stomata, elliptical guard cells, and smooth or erose stomatal rims. This was in consensus with a previous karyological study on Ligusticum, in which that no chromosomal differences detected between the two sections [11]. Furthermore, previous molecular analysis also showed that the species with entire bracteoles fell into two distant clades, and those with pinnate ones did not constitute a monophyletic group [18] [19]. Thus, this infrageneric subdivision provides a useful key for species identification, but is not a natural classification and is in need of further 
revision. Previous studies indicated that the pattern of anticlinal walls was associated with environmental factors [22] [28] [29] [30]. In our analysis, anticlinal walls in Ligusticum seems to be not related with their geographic distribution and environmental conditions where the species grow. For example, L. tenuissimum from northern China (growing on with rocky slopes) have straight to arched walls on both surfaces, while L. rechingerianum from southwest (on alpine meadows) also possess the same type.

\subsection{Relationships with Tilingia and Ligusticopisis}

The genus Ligusticopsis Leute has 14 species confined to China, all of which have been included in present study (note with ${ }^{*}$ in Table 1). Ligusticopsis was separated from Ligusticum based on the prominent calyx teeth of the former [4]. However, these prominent calyx teeth, actually occur in certain species of Ligusticum, e.g. L. involucratum Franch., which has conspicuous, triangular calyx teeth. Molecular analysis indicated that Ligusticopsis is not monophyletic, as species referred to Ligusticopsis fell into two distant clades, Selineae and Sinodielsia [18] [19]. In present study, epidermal characters including stomatal types, anticlinal walls and the cuticular membrane, could not differentiate Ligusticopsis from Ligusticum, as well as karyological evidence [11] and pollen morphology [31].

Tilingia Regel \& Tiling was established by Regel and Tiling [8], on the basis of distinct calyx teeth and the carpels bearing a solitary vitta in each furrow ( $T$. ajanensis Regel \& Tiling is the type species). However, this treatment was not accepted by Kozo-Poljansky [9] and Hiroe \& Constance [10], also following the pollen morphology experiments of Wang et al. [31]. In the present study, three species previously referred to Tilingia, L. ajanense (Regel \& Tiling) Koso-Poljansky, L. tachiroei (Franch. \& Savat.) Hiroe \& Constance and L. jeholense (Nakai \& Kitagawa) Nakai \& Kitagawa were included. L. ajanense and L. tachiroei are characterized by their anticlinal walls of adaxial and abaxial epidermis straight to arched and repand respectively, and the adaxial sides with no stomata. Morphologically, ribs on mericarps of these two species are prominent and subequal, which is differred from other species of the genus with winged lateral ribs. Previous molecular analysis showed that T. ajanensis allied with Pleurospermum hookeri C.B. Clarke, P. yunnanense Franch. and L. delavayi Franch. of the Acronema Clade, distantly related to other Ligusticum species [19]. Therefore, in accordance with the molecular and morphological results, epidermal evidence also supported the segregation of $L$. ajanense and L. tachiroei from Ligustium.

\subsection{The relationship between Hymenidium and Ligusticum delavayi}

As mentioned above, $L$. delavayi comprises a well-supported clade with Pleurospermum hookeri and P. yunnanense of the Acronema Clade. These taxa have similar fruit structures and membranous-margined bracts and all have been referred to genus Hymenidium Lindl. [32]. However, the other species referred to 
Hymenidium fell within tribe Pleurospermeae near the base of the trees, distant from Acronema Clade [19]. Therefore, Hymendium as currently circumscribed is not monophyletic. In this study, $L$. delavayi was quite different from other $L i$ gusticum species. For example, its epidermal cells were both irregular and rectangular, while cuticular membrane ornamentation was smooth. Furthermore, according to a previous study, the karyotype asymmetry of $L$. delavayi was the largest among species examined (T.C. $\%=58.53 \%$ ) [11]. In fact, with ultimate segments setuliform and membranous margined bracts, L. delavayi is distinct and easily differentiated from other Ligusticum species. Therefore, epidermal evidence, combined with molecular and karyological results, support that $L$. delavayi should be separated from Ligusticum.

\subsection{The Placement of $L$. peudodaucoides and L. moniliforme}

Ligusticum moniliforme and L. pseudodaucoides are two recently published taxa, which are not included by FOC. L. peudodaucoides was recorded only from the type locality, with its trinacriform bracteoles as the diagnostic character, and it most closely resembles $L$. daucoides (Franch.) Franch. [33]. However, its epidermal characters were different from that of $L$. daucoides (Table $2 \& \mathrm{Ta}$ ble 3). L. peudodaucoides distributed in Wuliangshan range of Yunnan Province, the southernmost geographical limit for the genus in China, while L. daucoides occurred in northern Yunnan and other provinces [2] [33]. L. moniliforme was described as a new species for its moniliform rhizome [34], however, L. nematophyllum (Pimenov \& Kljuykov) Pu \& Watson also has clustered fusiform or tuberous roots. Other characeters, e.g. leaf blade, bracteoles and petals are also very similar between the two species, as is the epidermal analysis under SEM. L. nematophyllum and L. moniliforme occur in western Sichuan and Gansu Province, respectively. Although our present analyses supply some leaf features for these two poorly known species (L. peudodaucoides and L. moniliforme), their relationships with each morphologically similar $L$. daucodes and $L$. nematophyllum needs more evidence.

\section{Conclusion}

In this study, a detailed observation of epidermal characters from across the genus Ligusticum is presented. Although not all species can be distinguished from each other by its own combinations of epidermal features, many characters seem to be important for distinguishing some closely related taxa. Furthermore, the previous infrageneric classification of $\mathrm{Pu}$ [1] is not supported by our present study, but the data do provide evidences for taxonomic treatment of some controversial taxa. In general, the results presented here will promote the future revisionary studies of this poorly known genus.

\section{Acknowledgements}

The authors thank two anonymous reviewers for helpful comments on the 
manuscript. This work was supported by the National Natural Science Foundation of China (No. 31460052) and the United Research Foundation of Yunnan Science and Technology Department-Kunming Medical University (No. 2015FB014).

\section{References}

[1] Pu, F.D. (1991) A Revision of the Genus Ligusticum L. (Umbelliferae) in China. Acta Phytotaxonomica Sinica, 29, 385-393.

[2] Pu, F.D. and Watson, M.F. (2005) Ligusticum L. In: Wu, Z.Y. and Raven, P.H., Eds., Flora of China, Vol. 14, Missouri Botanical Garden Press, St. Louis, 140-151.

[3] Drude, C.G.O. (1898) Umbelliferae. In: Engler, A. and Prantl, K., Eds., Die natürlichen Pflanzenfamilien, Vol. 3, Wilhelm Engelmann, Leipzig, Germany, 63-250.

[4] Leute, G.H. (1969) Untersuchungen über den Verwandtschaftskreis der Gattung Ligusticum L. (Umbelliferae). Teil I. Annalen des Naturhistorischen Museums in Wien, 73, 55-98.

[5] Thellung, A. (1926) Umbelliferae In: Hegi, G., Ed., Illustrierte Flora von Mitteleuropa, Vol. 5, Carl Hanser Verlag, München, 1415-1457.

[6] Schischkin, B.K. (1950) Flora URSS. Vol. 7, Akademii Nauk SSSR, Moscow.

[7] Leute, G.H. (1970) Untersuchungen über den Verwandtschaftskreis der Gattung Ligusticum L. (Umbelliferae). Teil II. Annalen des Naturhistorischen Museums in Wien, 74, 457-519.

[8] Regel, E. and Tiling, H. (1858) Florula ajanensis. Nouveaux Mémoires de la Société Impériale des Naturalistes de Moscou, 1, 97.

[9] Kozo-Poljansky, B.M. (1916) Sciadophytorum systematis lineamenta. Bulletin of Moscow Society of Naturalists, 29, 93-222.

[10] Hiroe, M. and Constance, L. (1958) Umbelliferae of Japan. University of California Publications in Botany, 30, 1-444.

[11] Zhou, J., Pu, F.D., Peng, H., Pan, Y.Z. and Gong, X. (2008) Karyological Studies of ten Ligusticum Species (Apiaceae) from the Hengduan Mountains Region of China. Caryologia, 61, 333-341. https://doi.org/10.1080/00087114.2008.10589644

[12] Downie, S.R. and Katz-Downie, D.S. (1996) A Molecular Phylogeny of Apiaceae Subfamily Apioideae: Evidence from Nuclear Ribosomal DNA Internal Transcribed Spacer Sequences. American Journal of Botany, 83, 234-251. https://doi.org/10.1002/j.1537-2197.1996.tb12701.x

[13] Plunkett, G.M., Soltis, D.E. and Soltis, P.S. (1996) Evolutionary Patterns in Apiaceae: Inferences Based on matK Sequence Data. Systematic Botany, 21, 477-495. https://doi.org/10.2307/2419610

[14] Katz-Downie, D.S., Valiejo-Roman, C.M., Terentieva, E.I., Troitsky, A.V., Pimenov, M.G., Lee, B. and Downie, S.R. (1999) Towards a Molecular Phylogeny of Apiaceae Subfamily Apioideae: Additional Information from Nuclear Ribosomal DNA ITS Sequences. Plant Systematics and Evolution, 216, 167-195. https://doi.org/10.1007/BF01084397

[15] Downie, S.R., Ramanath, S., Katz-Downie, D.S. and Llanas, E. (1998) Molecular Systematics of Apiaceae Subfamily Apioideae: Phylogenetic Analyses of Nuclear Ribosomal DNA Internal Transcribed Spacer and Plastid rpoC1 Intron Sequences. American Journal of Botany, 85, 563-591. https://doi.org/10.2307/2446441

[16] Downie, S.R., Katz-Downie, D.S. and Watson, M.F. (2000) A Phylogeny of the 
Flowering Plant Family Apiaceae Based on Chloroplast DNA rpl16 and rpoC1 Intron Sequences: Towards a Suprageneric Classification of Subfamily Apioideae. American Journal of Botany, 87, 273-292. https://doi.org/10.2307/2656915

[17] Valiejo-Roman, C.M., Shneyer, V.S., Samigullin, T.H., Terentieva, E.I. and Pimenov, M.G. (2006) An Attempt to Clarify Taxonomic Relationships in "Verwandtschaftskreis der Gattung Ligusticum" (Umbelliferae-Apioideae) by Molecular Analysis. Plant Systematics and Evolution, 257, 25-43.

https://doi.org/10.1007/s00606-005-0383-8

[18] Zhou, J., Peng, H., Downie, S.R., Liu, Z.-W. and Gong, X. (2008b) A Molecular Phylogeny of Chinese Apiaceae Subfamily Apioideae Inferred from Nuclear Ribosomal DNA Internal Transcribed Spacer Sequences. Taxon, 57, 402-416.

[19] Zhou, J., Gong, X., Downie, S.R. and Peng, H. (2009) Towards a More Robust Molecular Phylogeny of Chinese Apiaceae Subfamily Apioideae: Additional Evidence from nrDNA ITS and cpDNA Intron (rpl16 and rps16) Sequences. Molecular Phylogenetics and Evolution, 53, 56-68. https://doi.org/10.1016/j.ympev.2009.05.029

[20] Jones, J.H. (1986) Evolution of the Fagaceae: The Implications of Foliar Features. Annals of the Missouri Botanical Garden, 73, 228-275. https://doi.org/10.2307/2399112

[21] Baranova, M.A. (1992) Principles of Comparative Stomatographic Studies of Flowering Plants. The Botanical Review, 58, 49-99. https://doi.org/10.1007/BF02858543

[22] Stace, C.A. (1965). Cuticular Studies as an Aid to Plant Taxonomy. Bulletin of the British Museum (Natural History) Botany, 4, 1-78.

[23] Sharma, G.K. and Dunn, D.B. (1969) Environmental Modifications of Leaf Surface Traits in Datura stramonium. Canadian Journal of Botany, 47, 1211-1216. https://doi.org/10.1139/b69-171

[24] Dilcher, D. L. (1974) Approaches to the Identification of Angiosperm Leaf Remains. The Botanical Review, 40, 1-157. https://doi.org/10.1007/BF02860067

[25] Sun, N., He, X.-J. and Zhou, S.-D. (2010) Epidermal Characters of Ligusticum (Apiaceae) from China. Annales Botanici Fennici, 47, 261-279. https://doi.org/10.5735/085.047.0403

[26] Baranova, M.A. (1987) Historical Development of the Present Classification of Morphological Types of Stomates. The Botanical Review, 53, 53-79.

https://doi.org/10.1007/BF02858182

[27] Wilkinson, H.P. (1979) The Plant Surface (Mainly Leaf). In: Metcalfe, C.R. and Chalk, L., Eds., Anatomy of the Dicotyledons, 2nd Edition, Clarendon Press, Oxford, 97-167.

[28] Watson, R.W. (1942) The Effect of Cuticular Hardening on the Form of Epidermal Cells. New Phytologist, 41, 223-229. https://doi.org/10.1111/j.1469-8137.1942.tb07075.x

[29] Stace, C.A. (1984) The Taxonomic Importance of the Leaf Surface. In: Heywood, V.H. and Moore, D.M., Eds., Current Concepts in Plant Taxonomy, Academic Press, London, 67-94.

[30] Panteris, E., Apostolakos, P. and Galatis, B. (1994) Sinuous Ordinary Epidermal Cells: Behind Several Patterns of Waviness, a Common Morphogenetic Mechanism. New Phytologist, 127, 771-780. https://doi.org/10.1111/j.1469-8137.1994.tb02981.x

[31] Wang, P.L., Pu, F.D. and Ma, J.S. (1991) Pollen Morphology of the Genus Ligusticum from China and Its Systematic Significance. Acta Phytotaxonomica Sinica, 29, 235-245. 
[32] Pimenov, M.G. and Kljuykov, E.V. (2000) Taxonomic Revision of Pleurospermum Hoffm. and Related Genera of Umbelliferae III. The genera Physospemopsis and Hymenidium. Feddes Repertorium, 111, 535-552. https://doi.org/10.1002/fedr.20001110719

[33] Peng, H. and Wang, Y.Z. (1998) A New Species of Ligusticum L. (Apiaceae) from Yunnan Province, China. Novon, 8, 50-52. https://doi.org/10.2307/3391892

[34] Peng, Z.X. and Zhang, B.Y. (1995) A New Species of Ligusticum L. (Umbelliferae) from Gansu, China. Acta Phytotaxonomica Sinica, 33, 302-305. 


\section{Supplements}
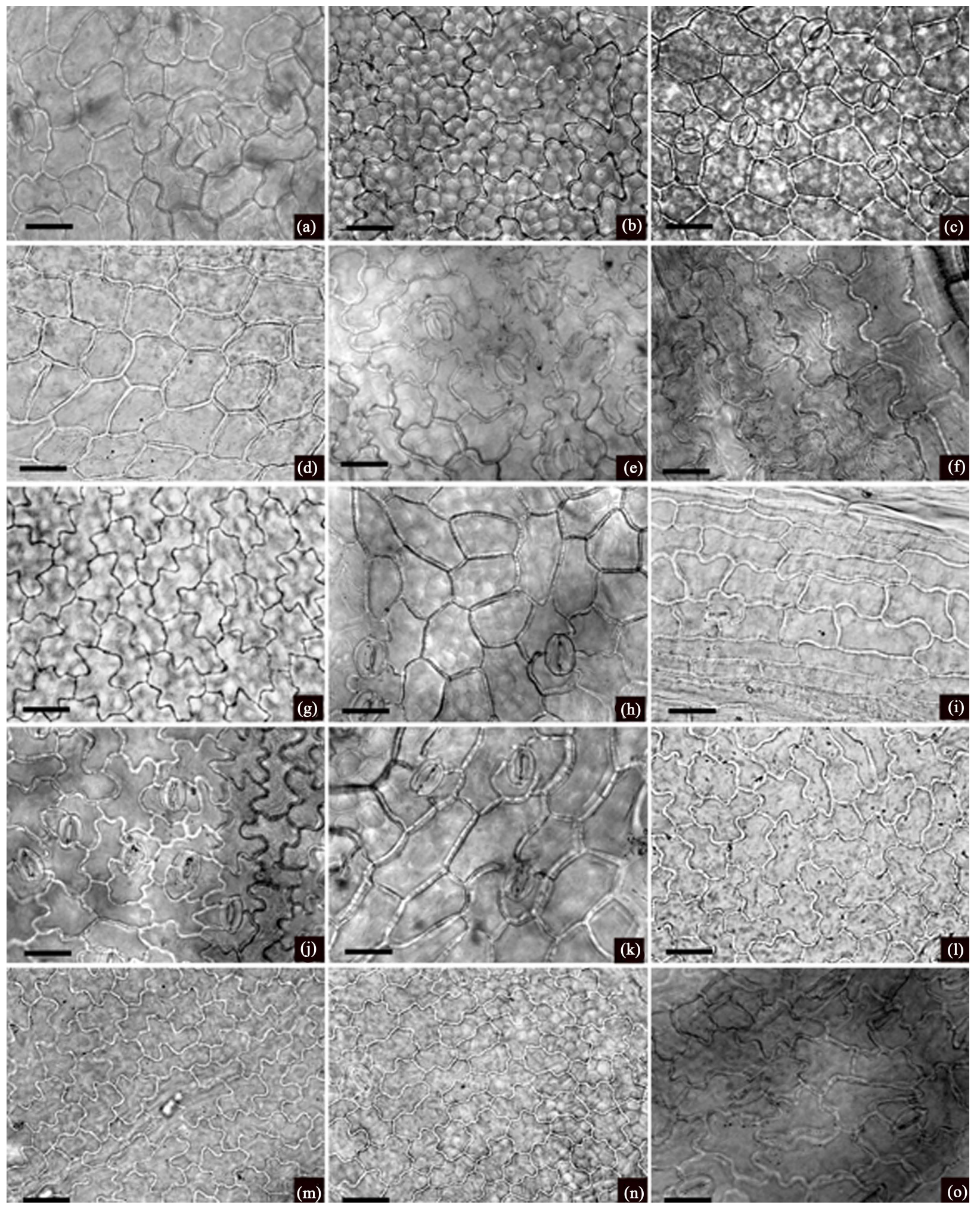

Figure S1. Characteristics of epidermal cells from Ligusticum species (light microscopy), all adaxial. (a) L. daucoides, (b) L. pteridophyllum; (c) L. litangense; (d) L. tachiroei; (e) L. hispidum. (f) L. nematophyllum; (g) L. sinense; (h) L. involucratum; (i) L. delavayi; (j) L. oliverianum; (k) L. likiangense; (l) L. littledalei; (m) L. acuminatum; (n) L. angelicifolium; (o) L. scapiforme. Scale bar $50 \mu \mathrm{m}$. 


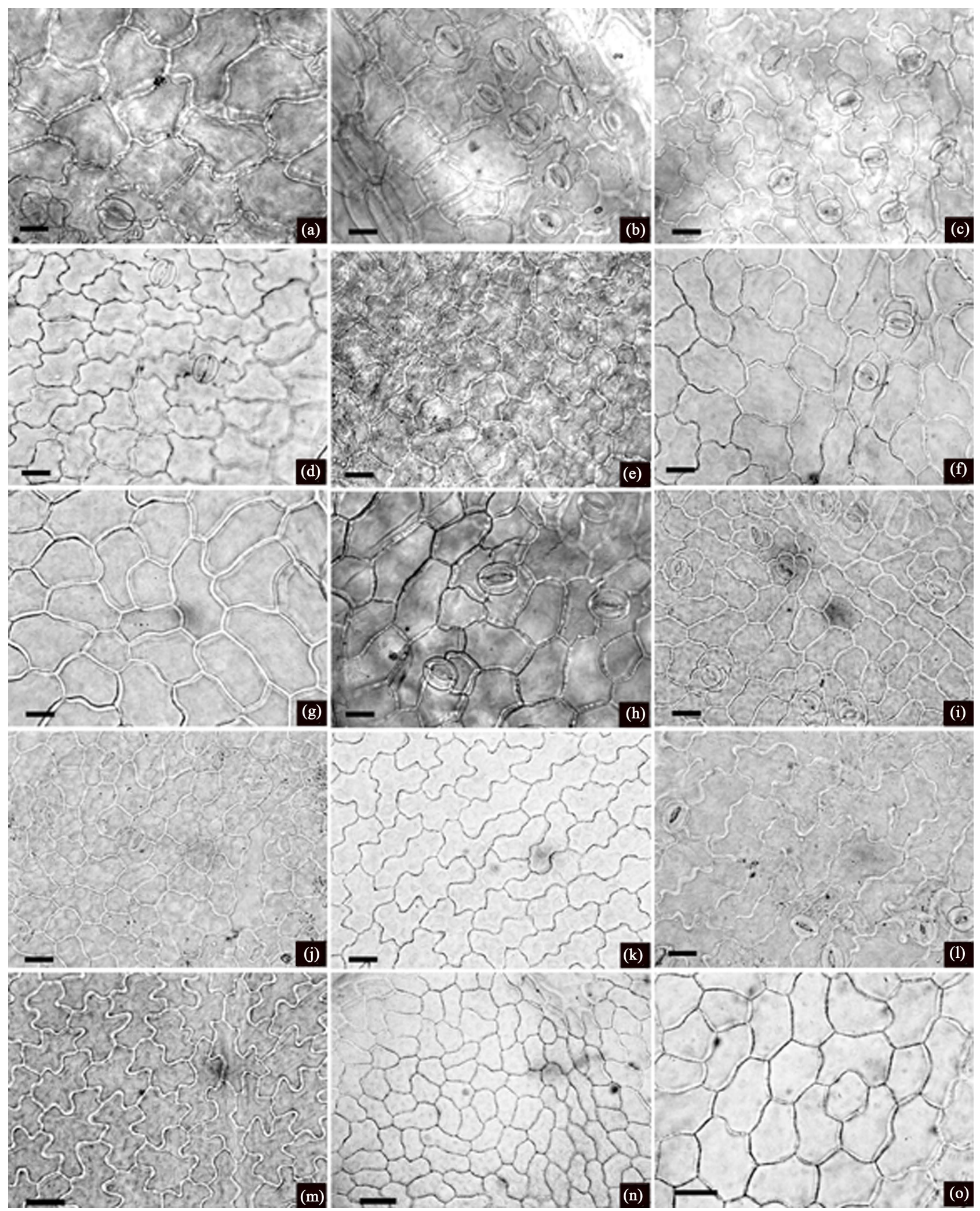

Figure S2. Characteristics of epidermal cells from Ligusticum species (light microscopy), all adaxial. (a) L. sikiangense; (b) L. mairei; (c) L. capillaceum; (d) L. multivittatum; (e) L. tenuisectum; (f) L. brachylobum; (g) L. tenuissimum; (h) L. yanyuanense. I. L. mucronatum; (j) L. thomsonii; (k) L. nullivittatum; (l) L. discolor; (m) L. jeholense; (n) L. weberbauerianum; (o) L. ajanense. Scale bar $50 \mu \mathrm{m}$. 


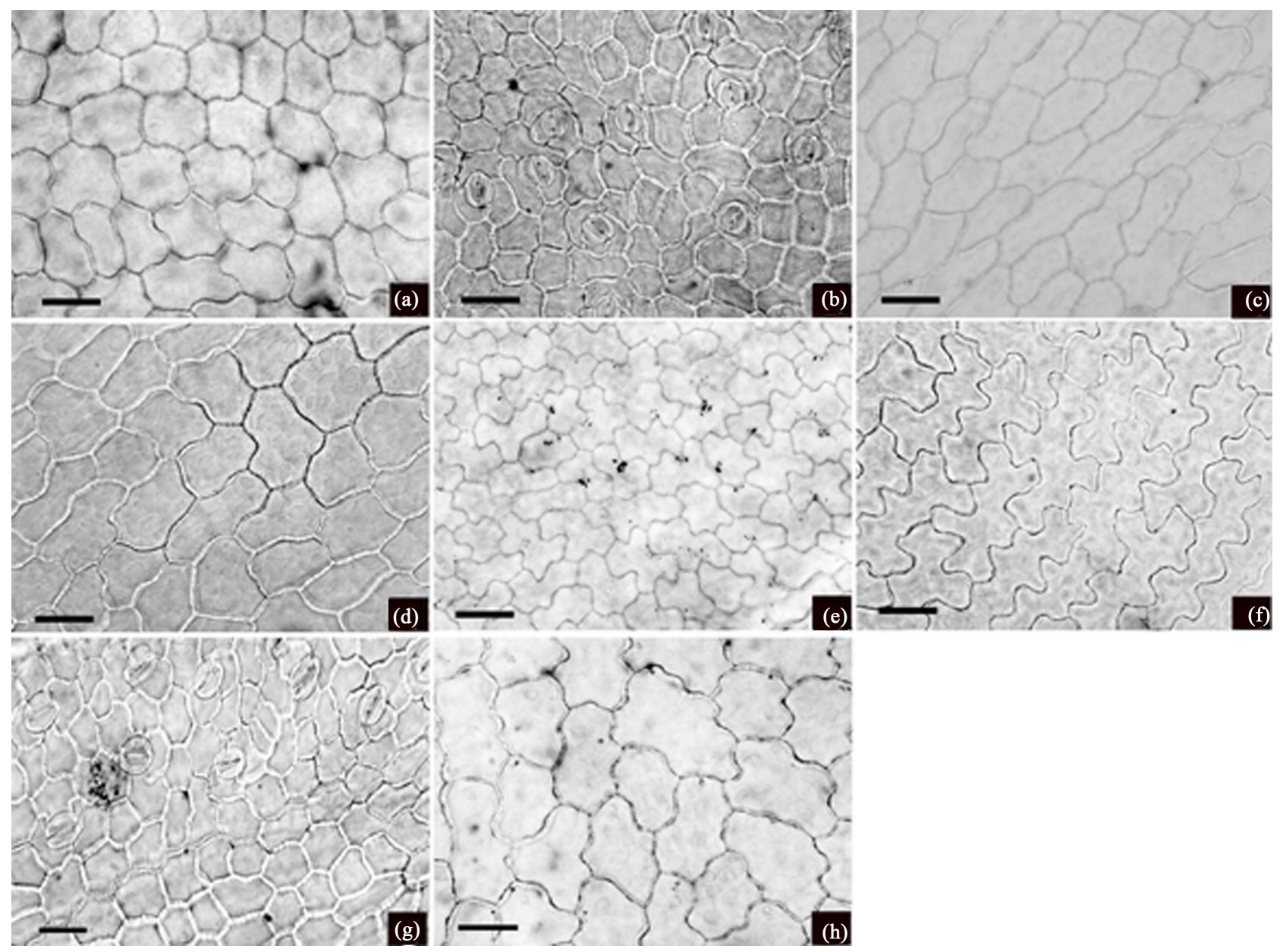

Figure S3. Characteristics of epidermal cells from Ligusticum species (light microscopy), all adaxial. (a) L. pseudodaucoides; (b) L. elatum; (c) L. reptans; (d) L. kingdon-wardii; (e) L. franchetii; (f) L. moniliforme; (g) L. rechingerianum; (h) L. sinense cv. Chuanxiong. Scale bar $50 \mu \mathrm{m}$. 